\title{
Mortality estimation for individual-based simulations of phosphine resistance in lesser grain borer (Rhyzopertha dominica)
}

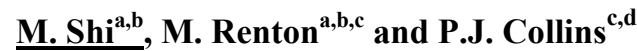 \\ ${ }^{a}$ M084, School of Plant Biology, FNAS, \\ The University of Western Australia, 35, Stirling Highway, Crawley, WA, 6009. \\ ${ }^{b}$ Cooperative Research Centre for National Plant Biosecurity, Australia \\ ${ }^{c}$ CSIRO Ecosystem Sciences, Underwood Avenue, Floreat, WA 6014, Australia \\ ${ }^{d}$ Department of Employment, Economic Development and Innovation, \\ Ecosciences Precinct, GPO Box 267 BRISBANE QLD 4001, Australia \\ Email: shi.mingren@gmail.com
}

\begin{abstract}
There is a world-wide need for the development of sustainable management strategies to control the development of phosphine $\left(\mathrm{PH}_{3}\right)$ resistance in lesser grain borer (Rhyzopertha dominica). Computer simulation models can provide a relatively fast, safe and inexpensive way to weigh the merits of various management options. However, the usefulness of simulation models relies on the accurate estimation of important model parameters.
\end{abstract}

Concentration and time of exposure are both important in determining the intensity of response to a toxic agent. The ability to estimate mortality or survival rate $(1$ - mortality) at a range of concentrations and exposure times based on experimental data is critical for the development of an accurate simulation of the evolution of resistance to phosphine. Our individual-based simulation model required predictions of finite daily survival rates at different concentrations for each of nine possible genotypes in our two-locus model.

In this paper we briefly described how we used a two-parameter probit model $Y=a+b \log (C t)$ and a fourparameter probit model $Y=a+b_{1} \log (t)+b_{2} \log (C)+b_{3} \log (t) \log (C)$ to fit three sets of experimental data (Collins et al, 2002, 2005; Daglish, 2004). Here $C(\mathrm{mg} / \mathrm{l})$ is the $\mathrm{PH}_{3}$ concentration, $t$ (hour) is the exposure time and $Y$ is the probit (= "probability unit") mortality, which is the probability of mortality transformed by the inverse cumulative distribution function (CDF) associated with the standard normal distribution.

The data sets of Collins et al. (2002, 2005) and Daglish (2004) are observed from five available strains of lesser grain borer, associated with five different genotypes. We still needed to construct a model predicting finite daily survival at different concentrations for the remaining four genotypes. As a step towards achieving this, we first estimated the resistance factors for the first five genotypes (strains) based on our fitted models. The resistance factor of a genotype $x$ for a given fumigation duration is defined as the ratio between the $\mathrm{PH}_{3}$ concentration that achieves $50 \%$ mortality in a sub-population of genotype $x$ and the lower $\mathrm{PH}_{3}$ concentration that achieves $50 \%$ mortality in a susceptible sub-population. We then estimated the resistance factors for the other four genotypes by making some basic assumptions regarding genetic interactions; log-transformed resistance factors for the nine genotypes can be expressed in terms of five parameters which represent respectively the strength and the dominance of the $1^{\text {st }}$ and $2^{\text {nd }}$ genes, and the synergism between the two genes. Finally we modelled survival rates for the other four genotypes using the two-parameter probit model. We assumed that the parameter $b$ for these genotypes was the same as the $b$ value for one of the five strains. Finally, the value for parameter $a$ for each of the four genotypes could then be obtained by direct substitution of the related values $C, t$ and $Y$ into the two-parameter probit model.

Having constructed the probit models we obtained estimates of finite daily survival rates in two ways with the same total survival rate for each of the nine genotypes.

Keywords: mortality estimation, probit models, finite daily survival rate, individual-based simulation, phosphine resistance, lesser grain borer 


\section{INTRODUCTION}

The lesser grain borer, Rhyzopertha dominica, is a very destructive primary pest of stored grains. Grain industries world-wide have come to rely heavily on phosphine $\left(\mathrm{PH}_{3}\right)$ fumigant for the control of infestations of the pest. This has led to the development of $\mathrm{PH}_{3}$ resistance in $R$. dominica in many countries, including Australia. Hence, there is a world-wide need for the development of sustainable resistance management strategies. We are currently developing a two-locus individual-based simulation model to predict the evolution of phosphine resistance in $R$. dominica, in order to weigh the merits of various management options for delaying or avoiding resistance.

Resistance to phosphine is an inherited trait. Our simulation model was constructed based on results from Collins et al. (2002) and Schlipalius et al. (2002). Their research revealed that the combination of alleles at two loci, rph1 and rph2, confers strong resistance and that $r p h 1$ by itself is responsible for the weak resistance phenotype. It seems that both rph1 and rph2 individually express a relatively low level of resistance but when they occur in the same insect the resistance mechanisms synergise, producing a much higher level of resistance. We consider two alleles on each of these two loci that govern the trait of resistance; dominant (susceptibility) alleles are denoted by $A$ and $B$, and recessive (resistance) by $a$ and $b$, respectively. Our two-locus simulation model includes nine possible genotypes:

- $\quad s s$ : with both loci homozygous susceptible $(A A$ and $B B)$

- $\quad s h$ : with the first locus homozygous susceptible $(A A)$ and the second locus heterozygous $(B b)$

- $\quad s r$ : with the first locus homozygous susceptible $(A A)$ and the second locus homozygous resistant $(b b)$

- $\quad$ and similarly $h s, h h, h r, r s, r h$, through to

- $\quad r r$ : with both loci homozygous (strongly) resistant ( $a a$ and $b b$ ).

Computer simulation models can provide a relatively fast, safe and inexpensive way to project the consequences of different assumptions about resistance and to weigh the merits of various management options. But the usefulness of such models depends on generating or estimating the values of key parameters. In our individual-based model, these parameters include mortalities of insect pests under various pesticide doses, the chance of certain genotypes being produced as the result of the mating of certain parent genotypes (which we call offspring genotype tables), initial frequencies of genotypes, and the intrinsic rate of natural increase of an insect population. These are important parameters within the sub-models for simulating genetic recombination and thus determining the genotype of offspring, initialisation of the population, simulating the effects of pesticide applications and calculating the number of eggs produced by each insect, respectively. The ability to estimate mortality for all possible genotypes at a range of concentrations and expose times based on experimental data is particularly critical for the development of an accurate simulation of the evolution of resistance to phosphine. In this paper, we describe how we developed a series of empirical models to achieve this for our simulation model.

First we fitted probit model of mortality for five genotypes, for which mortality data was available, as described in Section 2. We still needed to construct a model predicting survival rates at different concentrations for the remaining $s h, s r, h r$ and $r h$ genotypes. As a step towards achieving this, we first estimated the resistance factors for the first five genotypes, as described in Section 3. We then estimated the resistance factors for the other four genotypes ( $s h, s r, h r$ and $r h$ ) (Section 4). This was done by making some plausible assumptions regarding genetic interactions. We modelled survival rates for these genotypes using equation $Y=a+b \log (C t)$, as described in Section 5. Finally we describe how we estimated finite daily survival rate of each genotype for our simulations using probit models in Section 6. As our individual-based simulation is based on a discrete daily time-step, we obtain an estimate for the finite daily survival rate in two ways with the same total survival rate for each genotype.

\section{FITTING PROBIT MODELS OF MORTALITY FOR FIVE STRAINS}

We used a novel method, least squares technique with generalized inverse matrix approach (e.g. Ben-Israel and Greville, 2003), to fit available data (Shi and Renton, 2011). This method has advantages over other methods: it is simple with only one key command, provides a more accurate estimate of parameters, and even if the coefficient matrix of the over-determined linear system is not numerically (column) full ranked it will still work and yield a solution with minimum error in the $L_{2}$ norm sense (Ben-Israel and Greville, 2003).

Collins et al. (2002) observed mortalities under a range of concentrations ( $C: \mathrm{mg} / \mathrm{l})$ of phosphine at exposure time $(t) 48 \mathrm{hr}$ for susceptible (strain QRD14 - corresponding to genotype ss) and strong resistant (strain QRD569 - rr $)$ phenotypes and their combined $\mathrm{F}_{1}$ progeny $((569 \times 14)+(14 \times 569)-h h)$. The neatened raw data 
are listed in Table 1. Note that if the response (kill) rate is $p=$ 1 or 0 then we should change it from 1 to $\mathbf{0 . 9 9 9 9}$ or from 0 to $\mathbf{0 . 0 0 0 1}$ or $\mathbf{1 0}^{-\mathbf{8}}$. Otherwise its probit value is undefined.

The experiments in Collins et al. (2005) were done over a long period of time and the results were confirmed in field trials and are the basis for the current rates used to control resistant insects. $L T_{99.9}$ values (lethal time to achieve 99.9\% mortality) derived from their observed data for strain QRD569 exposed to a series of fixed concentration from 0.1 to $1.0 \mathrm{mg} / \mathrm{l}$ at a range of exposure periods are listed in the $1^{\text {st }}$ and $2^{\text {nd }}$ rows of Table 2 .

Daglish (2004) determined mortality rates of $50 \%\left(L C_{50}\right)$ and $99 \%\left(L C_{99}\right)$ for phosphine-susceptible (QRD14 - $\left.s s\right)$ and weak-resistance $(\mathrm{QRD} 369-r s)$ phenotypes and their F1 progeny $(\mathrm{QRD} 369 \times \mathrm{QRD} 14-h s)$ over a range of concentrations and exposure times (Table 3)

We used the two-parameter probit model to predict mortalities for genotypes ss and $h h$ using data in Table 1:

$Y=a+b \log (C t)$.

Here $Y$ is the probit (= "probability unit") mortality, the inverse cumulative distribution (CDF) value plus 5 associated with the standard normal distribution. For example, for 0.5 or $50 \%$ mortality, the inverse CDF value is 0 (or when the random variable equal to the mean value 0 , the $\mathrm{CDF}$ value is 0.5 ) and the probit value $Y=$ 5.

Daglish (2004) used a Haber-type model (Bunce and Remillard, 2003), $C^{n} t=k$, to predict response to phosphine, for example, $C^{0.8673} t=0.2088$ for the susceptible strain QRD14 at the $L C_{50}$ (see Table 3). As $n$ and $k$ in their equations vary with genotype and mortality, it is not possible to develop a Habertype rule with which to successfully Table 3. Concentration $\left(L C_{50} \& L C_{99}\right)$ values $(\mathrm{mg} / \mathrm{l})$ required to achieve $50 \%$ and $99 \%$ mortality for different exposure times $(t)$ (Daglish (2004))

\begin{tabular}{|l|c|c|c|c|c|c|}
\hline Strain & Mortality & $20 \mathrm{~h}$ & $48 \mathrm{~h}$ & $72 \mathrm{~h}$ & $144 \mathrm{~h}$ & $C^{n} t=k$ \\
\hline QRD14 & $50 \%$ & 0.0052 & 0.0017 & 0.0011 & 0.00064 & $C^{0.8673} t=0.2008$ \\
$(s s)$ & $99 \%$ & 0.0091 & 0.0037 & 0.0021 & 0.0014 & $C^{0.8540} t=0.4108$ \\
\hline QRD369 & $50 \%$ & 0.20 & 0.052 & 0.032 & 0.017 & $C^{0.8673} t=4.0908$ \\
$(r s)$ & $99 \%$ & 0.40 & 0.091 & 0.060 & 0.028 & $C^{0.8540} t=7.0783$ \\
\hline QRD369X & $50 \%$ & 0.010 & 0.0042 & 0.0023 & 0.0011 & $C^{0.8673} t=0.3863$ \\
QRD14 $(h s)$ & $99 \%$ & 0.026 & 0.013 & 0.0066 & 0.0025 & $C^{0.8540} t=0.9715$ \\
\hline
\end{tabular}

Table 4. (a) Parameters derived from data of Collins et al. (2002) fitted to two-parameter model (1)

\begin{tabular}{|l|c|c|}
\hline Strain & $a$ & $b$ \\
\hline QRD14 (ss) & 15.032386 & 9.229083 \\
\hline QRD569×14 & 7.954711 & 5.913329 \\
\hline
\end{tabular}

(b) Parameters derived from data of Daglish (2004) with Collins et al. $(2002,2005)$ fitted to four-parameter model (2)

\begin{tabular}{|l|r|r|r|r|}
\hline Strain & \multicolumn{1}{|c|}{$a$} & $b_{1}$ & $b_{2}$ & $b_{3}$ \\
\hline QRD14 $(s s)$ & 3.974876 & 12.326743 & 3.870027 & 1.924693 \\
\hline QRD369×14 $(h s)$ & 11.284676 & 3.776399 & 6.964954 & -1.010451 \\
\hline QRD369 $(r s)$ & -10.413046 & 15.575413 & 0.047656 & 4.701759 \\
\hline QRD569 $(r r)$ & -12.232356 & 10.386287 & 3.101974 & 1.190773 \\
\hline
\end{tabular}
extrapolate predicted mortalities between exposure scenarios (Bunce and Remillard, 2003). Considering the interaction of concentration and exposure time, however, we employed the four-parameter probit model to refit their data: (incorporated with $L T_{99.9}$ value at $C=0.02 \mathrm{mg} / \mathrm{l}$ and $t=352.8 \mathrm{~h}$ for the strain QRD369) 
Shi et al., Mortality estimation for simulations of phosphine resistance in $R$. dominica

$Y=a+b_{1} \log (t)+b_{2} \log (C)+b_{3} \log (t) \log (C)$

This model was also used to fit the parts of both data sets of Collins et al. $(2002,2005)$ at $C$ from 0.1 to $1.0 \mathrm{mg} / \mathrm{l}$. The parameters for the five strains are listed in Table 4. Details are included in Shi and Renton (2011). Details of probit models are included in Finney (1971).

\section{ESTIMATION OF RESISTANCE FACTORS FOR THE FIVE STRAINS}

The resistance factor of a genotype $x$ for a given fumigation duration is defined as the ratio between the $\mathrm{PH}_{3}$ concentration that achieves $50 \%$ mortality in a subpopulation of genotype $x$ and the lower $\mathrm{PH}_{3}$ concentration that achieves $50 \%$ mortality in a susceptible sub-population (FAO, 1975). Mathematically, the resistance factor for genotype $x$ is yielded by

$$
f(x)=L C_{50}(x) / L C_{50}(s s) .
$$

We estimated $L C_{50}$ for 48 hour exposure for the first five genotypes using our fitted models (1) or (2) by inserting $t=48$ and probit value $Y=5$ (corresponding to 0.5 or $50 \%$ mortality). The results are listed in Table 5 [reported by Daglish (marked by \#D) and Collins et al. (marked by \#C) and estimated by Eq. (2) or (1)]. It can be see that the estimated $L C_{50}$ values obtained using Eq. (1) and (2) are very close to reported values for all strains except for the $h h$ genotype. In addition, estimated $L T_{99.9}$ values are all very close to the observed $L T_{99.9}$

Table 6. The expressions of log-transformed resistance factors $f(x)$ for genotype $x$ in terms of five parameters

\begin{tabular}{|c|c|c|c|}
\hline $\begin{array}{l}2^{\text {nd }} \text { gene } \\
1^{\text {st }} \text { gene }\end{array}$ & $s$ & $h$ & $r$ \\
\hline$s$ & 0 & $\begin{array}{l}d_{2} s_{2} \\
{\left[=x_{2}=\ln f(s h)\right]}\end{array}$ & $s_{2}[=\ln f(s r)]$ \\
\hline$h$ & $d_{1} s_{1}$ & $\begin{array}{l}\boldsymbol{d}_{2} \boldsymbol{s}_{2}+d_{1} \boldsymbol{s}_{1}+\boldsymbol{k}\left(d_{1} \mathbf{s}_{1}\right)\left(d_{2} \mathbf{s}_{2}\right) \\
{[=\ln f(h h)]}\end{array}$ & $\begin{array}{l}d_{1} s_{1}+s_{2}+k\left(d_{1} s_{1}\right) s_{2} \\
{\left[=x_{1}=\ln f(h r)\right]}\end{array}$ \\
\hline$r$ & $s_{1}$ & $\begin{array}{l}d_{2} s_{2}+s_{1}+k s_{1}\left(d_{2} s_{2}\right) \\
{\left[=x_{3}=\ln f(r h)\right]}\end{array}$ & $\begin{array}{l}\boldsymbol{s}_{2}+\boldsymbol{s}_{1}+\boldsymbol{k} \boldsymbol{s}_{1} \boldsymbol{s}_{2} \\
{[=\ln f(r r)]}\end{array}$ \\
\hline
\end{tabular}
values (Table 2). This means our estimated resistance factors (Table 7) are also very close to the corresponding reported values. Note that the $L C_{50}$ values of the susceptible genotype ( $s s$ ) reported by the two papers are very close to each other: 0.00169 and 0.00174 , and they are the same to four decimal places: 0.0017 which is also the same as our estimated value. Hence in what follows we only use model (1) to predict mortalities for the ss genotype.

\section{ESTIMATION OF RESISTANCE FACTORS FOR THE OTHER FOUR GENOTYPES}

We estimated the resistance factors for the other four genotypes ( $s h, s r, h r$ and $r h$ ) by making some basic plausible assumptions regarding genetic interactions. We assumed that log-transformed resistance factors for the nine genotypes can be expressed in terms of five parameters $k, d_{1}, d_{2}, s_{1}$ and $s_{2}$ as shown in Table 6 . Here $s_{1}$ and $s_{2}$ represent the strength of the $r p h 1$ and $r p h 2$ genes respectively, $d_{1}$ and $d_{2}$ represent the dominance of the $r p h 1$ and $r p h 2$ genes respectively, and $k$ represents the synergism between the two genes.

For the simplicity of notation we defined the constants

$C_{h s}=\ln f(h s)$,

$C_{h h}=\ln f(h h)-\ln f(h s)$,

$C_{r r}=\ln f(r r)-s_{1}$.

Then we have the following four equations with five unknowns $\left(x_{1}, x_{2}, x_{3}, s_{2}\right.$ and $k$ ) from the expressions for the genotypes $r r, h r, h h$, and rh from Table 6:
Table 7. The reported $(\log ) L C_{50}$ factors for the five strains and the estimated $L \mathrm{C}_{50}$ factors for the remaining four genotypes $s h, s r, h r$ and $r h$ obtained from the relationships listed in Table 6 .

\begin{tabular}{|c|l|l|l|}
\hline $\begin{array}{c}2^{\text {nd }} \text { gene } \\
1^{\text {st }} \text { gene }\end{array}$ & \multicolumn{1}{|c|}{$s$} & \multicolumn{1}{c|}{$h$} & \multicolumn{1}{c|}{} \\
\hline$s$ & 0 & $\begin{array}{l}d_{2} s_{2}=\ln f(s h)=x_{2} \\
* f(s h)=1.2537\end{array}$ & $\begin{array}{l}s_{2}=\ln f(s r) \\
* f(s r))=5.5307\end{array}$ \\
\hline$h$ & $f(h s)=2.4706$ & $f(h h)=3.2235$ & $d_{1} s_{1}+s_{2}=\ln f(h r)=x_{1}$ \\
& $d_{1} s_{1}=\ln f(h s)=0.9045$ & $\ln f(h h)=1.1705$ & $* f(h r)=18.4839$ \\
\hline$r$ & $f(r s)=30.5882$ & $d_{2} s_{2}+s_{1}=\ln f(r h)=x_{3}$ & $f(r r)=530.3788$ \\
& $s_{1}=\ln f(r s)=3.4206$ & $* f(r h)=44.6005$ & $\ln f(r r)=6.2736$ \\
\hline
\end{tabular}




$$
\begin{aligned}
& \left(s_{1} k\right) s_{2}+s_{2}=C_{r r}(\text { from } r r), x_{1}-C_{h s}\left[s_{2} k\right]-s_{2}=C_{h s}(\text { from } h r), \\
& C_{h s}\left[k x_{2}\right]+x_{2}=C_{h h}(\text { from } h h), x_{3}-s_{1}\left[k x_{2}\right]-x_{2}=s_{1}(\text { from } r h) .
\end{aligned}
$$

We already had estimates for the factors $f(s s), f(h s), f(r s), f(h h)$ and $f(r r)$. From experimental data we also knew $f(s r)$ is less than $f(r s)$, and so $s_{2}<s_{1}$. We therefore assumed that $s_{2}[=\ln f(s r)]=p s_{1}$, with $p=0.5$, which gave an estimate for $f(s r)$, and then solved for the remaining unknowns successively from Eq. (5) to give estimates for $f(s h), f(r h)$ and $f(h r)$ :

$\begin{aligned} k & =\left(C_{r r}-p s_{1}\right) / p s_{1} s_{1}, x_{1}=C_{h s}+k p s_{1} C_{h s}+p s_{1} \\ x_{2} & =C_{h h} /\left(k C_{h s}+1\right), x_{3}=\left(k s_{1}+1\right) x_{2}+s_{1} .\end{aligned}$

The $L C_{50}$ resistance factors reported for the five strain and estimated for the other four genotypes can be derived from (see Eq.(3) and Table 5)

$$
L C_{50}(x)=0.0017 f_{50}(x) .
$$

The results are listed in Table 7 . Note that the estimated (ln) $L C_{50}$ factors for rr genotype were $f(r r)=602.9418$ and $\ln f(r r)=6.4018$ respectively if we used Collins et al. (2002) data only to fit the probit model (1).

\section{CONSTRUCTING A PROBIT MORTALITY MODEL FOR THE OTHER FOUR GENOTYPES}

Fig. 1 shows the plot of probit values based on our fitted models against a range of concentration values $[0.01,1.0](\mathrm{mg} / \mathrm{l})$ at a fixed $t=24$ hrs for $s s, h h$ and $r r$ predicted from Eq. (1) and for $h s$ and $r s$ genotypes from Eq. (2). Note this is different to the finite daily rate we discuss below.

For modelling survival rates using Eq. (1) for the other four genotypes ( $x=s h, s r, h r$ and $r h)$, we needed to fix two parameters $a(x)$ and $b(x)$. We see from Fig. 1 that the middle three lines for the $h s, h h$ and $r s$ genotypes are nearly parallel implying that the slopes are nearly the same. We assumed that the parameter $b(x)$ for these genotypes is the same as the estimated slope for the $h h$ genotype, $b(h h)$. Under this assumption it can be expected that none of the nine probit lines (at a fixed time $t=24 \mathrm{hrs}$ ) would intersect within the "middle zone" $(-1.706<Y<11.706)$. We already had estimates for the resistance factors for $t=48 \mathrm{hrs}$ for each of the four genotypes. We obtained estimates of their $L C_{50}$ values by the formula (7). Furthermore, we estimate the parameter $a(x)$ for $x=s h, s r, h r$ and $r h$ using model (1) with all $b(x)=b(h h)=5.913329, \log (C)=\log \left(L C_{50}(x)\right)$ and $Y(x) \equiv 5$ (mortality $50 \%$ ). The estimated parameters $a(x)$ are as follows:

$$
a(s h)=10.854928, a(s r)=7.043248, a(h r)=3.944565 \text {, and } a(r h)=1.682448 .
$$

\section{ESTIMATION OF FINITE DAILY SURVIVAL RATE}

Now we had a model predicting survival rates at a range of concentrations and times for each of the nine genotypes. For the $h s$ and $r s$ genotypes this model was of the form of Eq. (2) and for the other genotypes it was of the form of Eq. (1). The probit lines at $t=24 \mathrm{hrs}$ for all of the nine genotypes are shown in Fig. 2. Note that although the two lines for genotypes $r s$ and $r h$ intersect at the point $(C, Y)=(0.0103,-1.91363)$. 
This point is outside the "middle zone" which means both of these genotypes will effectively have $100 \%$ survival at such a low dose.

Fig. 3 (a) shows the survival rates for genotypes $s s, s h, s r, h s$ and $h h$ at $t=24 \mathrm{hrs}$ and the range of $C$ from 0.01 to $0.05 \mathrm{mg} / \mathrm{l}$ and (b) shows those for genotypes $h h, h r, r s, r h$ and $r r$ at $t=24 \mathrm{hrs}$ and the range of $C=$ $[0.01,1.0]$. The survival curve for genotype $h h$ appears in (a) and (b) for the purpose of comparison. Note different $x$-axis scales and the survival rate at $C=1.0 \mathrm{mg} / \mathrm{l}$ is about $99.81 \%$.

(a)

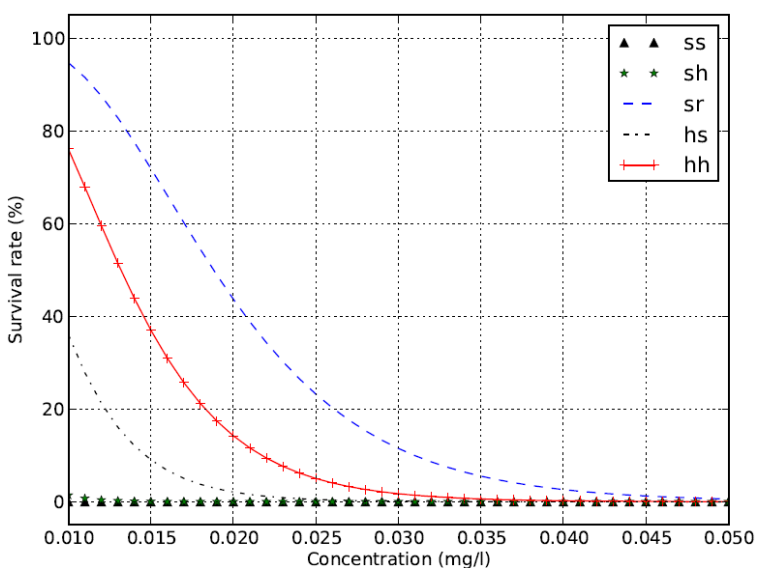

(b)

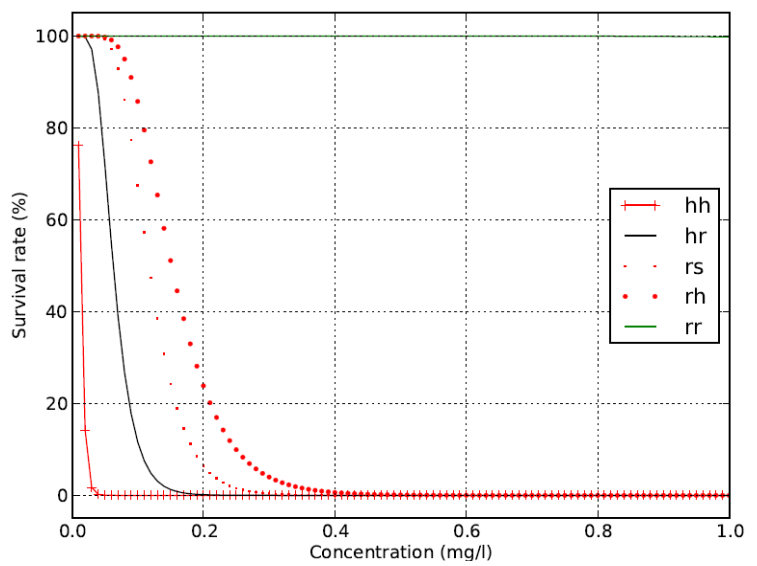

Figure 3. Survival rates by substituting $t=24 \mathrm{hrs}$ and a range of $C$ values for (a) genotypes $s s, s h, s r, h s$ and $h h$ and (b) genotypes $h h, h r, r s, r h$ and $r r$. Note different $x$-axis

There are two ways to estimate the finite daily survival rate (FDSR) under a $\mathrm{PH}_{3}$ treatment $C \times T$ at a fixed concentration $C(\mathrm{mg} / \mathrm{l})$ and a range of times $(1,2, \ldots, T$ days). Directly substituting the fixed $C$ value and the series of $t$ values into the prediction Eqs (1) and (2) results in the cumulative survival rates (CSR). The cumulative survival rate for the final $T^{\text {th }}$ day is the total survival rate $S V_{T}(x)$ for genotype $x$ for the full $C \times T$ treatment. One way to estimate FDSR is based on the assumption that the survival rate is the same each day during a fumigation treatment, in which case the FDSR is $S V_{s 1}(x)=\left[S V_{T}(x)\right]^{1 / T}$. The estimated finite daily and total survival rates under the two treatments of $0.01 \mathrm{mg} / \mathrm{l} \times 14$ days and $0.2 \mathrm{mg} / \mathrm{l} \times 8$ days are listed in Tables 8 and 9 . The other way to estimate different FDSR for each day is as follows: convert the $\mathrm{CSR}_{i+1}$ and $\mathrm{CSR}_{i}$ into the $i^{\text {th }}$ day's daily survival rate $S V_{d i}(x)$ by setting $S V_{d 1}(x)=\operatorname{CSR}_{1}(x)$ and then letting $S V_{d i}(x)=\operatorname{CSR}_{i+1}(x) / \operatorname{CSR}_{i}(x), i=1,2, \ldots, T-1$. Note that the total survival rate obtained by either of the two ways will be the same for each genotype. Fig. 4 shows the FDSR and CSR values for $r r$ beetle obtained from the two ways. If the simulation is only concerned with the results after fumigation, then we can use the former approach with equal daily survival rates, as it is simpler. If the simulation is also concerned with accurately representing what happens during the fumigation process then we should use the latter approach, even though it is more complex and will increase the simulation time.

\section{DISCUSSION AND CONCLUSION}

Note that the predicted survival rates depend on the prediction model and the observed data. Here the treatment $0.2 \mathrm{mg} / \mathrm{l} \times 8$ days was predicted to kill $99.2 \%$ of strongly-resistant beetles and to eradicate the other genotypes of beetles (their predicted survival rates after 9 days fumigation are all less than $1 . \times 10^{-11}$ ).

We also constructed another individual-based model which is based on the simplifying assumption that resistance is conferred by alleles at a single locus. When we compared the differences between the one- and 
two-locus models under the two treatments above, we aggregated the nine genotypes from the twolocus model into just three groups each corresponding to a one-locus genotype. Then we set the survival rates for the three genotypes in the one-locus model as the mean value of the survival rates of the group elements. Hence the results described here provided survival rates for the onelocus model as well.

Using the two-locus model, we are currently investigating some operational factors which influence the development of phosphine resistance: the impact of different life stages and the impact of different initial gene frequencies on the effect of fumigation. All the finite daily survival rates for these studies are predicted by probit models (1) and (2) for the nine genotypes, as described in this paper.

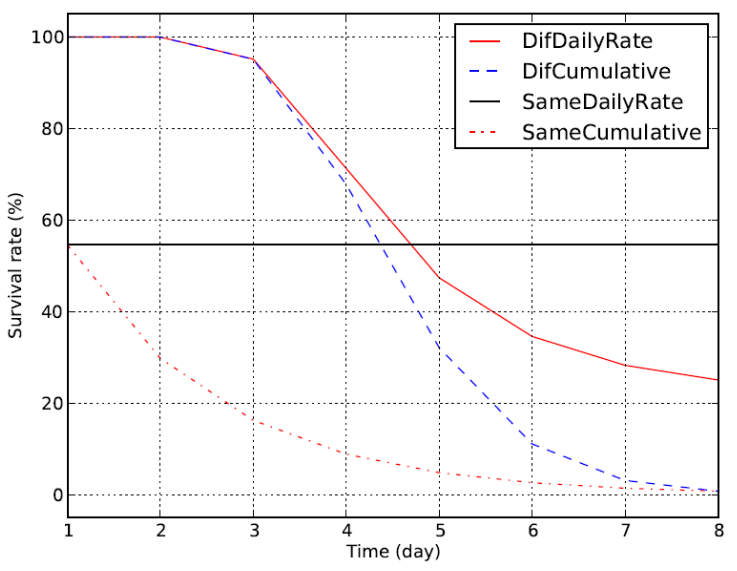

Figure 4. The same/different daily and cumulative survival rate for $r r$ beetle under $0.2 \mathrm{mg} / 1 \times 8$ days treatment

The results described in this paper allow us to accurately predict morality of the nine resistance genotypes of the lesser grain borer, Rhyzopertha dominica. This provides an essential component of our two-locus individual-based simulation model, which will help us predict the evolution of phosphine resistance in $R$. dominica, in order to weigh the merits of various management options for delaying or avoiding evolution of resistance in this destructive primary pest of stored grains. Since the project includes industry researchers and is supported by the National Plant Biosecurity CRC, which includes many collaborators from the storedgrains industry, recommendations resulting from this study will have a clear route to extension and adoption. This will allow us to continue to use the relatively safe and effective phosphine $\left(\mathrm{PH}_{3}\right)$ fumigant for the control of infestations of this serious pest, and thus help safeguard world-wide grain supplies.

\section{ACKNOWLEDGMENTS}

The authors would like to acknowledge the support of the Australian Government's Cooperative Research Centres Program. We also thank Rob Emery, James Ridsdill-Smith and Yonglin Ren for their great help in provision of raw data and information about beetle life cycles and silo fumigation.

\section{REFERENCES}

Ben-Israel, A., Greville, T.N.E., (2003). Generalized Inverses: Theory and Applications, $2^{\text {nd }}$ ed. New York, NY: Springer.

Bunce N.J., Remillard, R. B., (2003). Haber's rule: The search for quantitative relationships in toxicology. Human and Ecological Risk Assessment, 9(B.10), 973-985.

Collins, P.J., Daglish, G.J., Bengston, M., Lambkin, T.M., Pavic, H., (2002). Genetics of resistance to phosphine in Rhyzopertha dominica (Coleoptera: Bostrichidae). Journal of Economic Entomology 95, 862-869.

Collins, P. J., Daglish, G. J., Pavic, H., Kopittke, R. A., 2005. Response of mixed-age cultures of phosphineresistant and susceptible strains of lesser grain borer, Rhyzopertha dominica, to phosphine at a range of concentrations and exposure periods. Journal of Stored Products Research 41, 373-385.

Daglish, G. J., (2004). Effect of exposure period on degree of dominance of phosphine resistance in adults of Rhyzopertha dominica (Coleoptera: Bostrychidae) and Sitophilus oryzae (Coleoptera: Curculionidae). Pest Management Science 60 (2), 822-826.

Finney, D.J., (1971). Probit Analysis, Cambridge University Press, $3^{\text {rd }}$ edition.

Schlipalius, D.I., Cheng, Q., Reilly, P.E., Collins, P.J., Ebert, P.R., 2002. Genetic linkage analysis of the lesser grain borer Rhyzopertha dominica identifies two loci that confer high-level resistance to the fumigant phosphine. Genetics Society of America 161, 773-782.

Shi, M., Renton, M., (2011). Numerical algorithms for estimation and calculation of parameters in modelling pest population dynamics and evolution of resistance in modelling pest population dynamics and evolution of resistance. Mathematical Biosciences, http://dx.doi.org/10.1016/j.mbs.2011.06.005 